\title{
LOGARITHMIC COMPLEMENTARY MEANS AND AN EXTENSION OF CARLSON'S LOG
}

\author{
Peter Kahlig and Janusz Matkowski
}

Abstract. The invariance equality $L \circ(\mathscr{M}, \mathscr{N})=L$, where $L$ is the logarithmic mean, and where the unsymmetric compound means $\mathscr{M}=A \circ\left(P_{1}, G\right), \mathscr{N}=A \circ\left(P_{2}, G\right)$ are built with the arithmetic $A$, geometric $G$, and projective means $P_{1}, P_{2}$, is called "Carlson's log" and is important in iteration of means. In the present paper we present effective and simple 1 -parameter families of unsymmetric means $M_{t}, N_{t}:(0, \infty)^{2} \rightarrow(0, \infty)$ such that, for all $t \in(-1,1)$,

$$
L \circ\left(M_{t}, N_{t}\right)=L
$$

and

$$
\mathscr{M}=M_{\frac{1}{2}}, \quad \mathscr{N}=N_{\frac{1}{2}} .
$$

Existence of elementary (simple) symmetric means $M$ and $N$ such that $L \circ(M, N)=L$ and $M \neq L$ is posed as an open problem.

Mathematics subject classification (2010): Primary: 26A17, 26E60, 39B22.

Keywords and phrases: Mean, symmetric mean, mean-type mapping, iteration, invariant mean, Gaussian product, complementary means, Carlson's log.

\section{REFERENCES}

[1] H. Alzer, S. Ruscheweyh, On the intersection of two-parameter mean value families, Proc. Am. Math. Soc. 129 Nr. 9 (2001), 2655-2662.

[2] J. M. Borwein, P. B. Borwein, Pi and the AGM, A Wiley-Interscience Publication, New York/Chichester/Brisbane/Toronto/Singapore, 1987.

[3] P. S. Bullen, Handbook of means and their inequalities, Kluwer Academic Publishers, Dordrecht/Boston/London, 2003.

[4] B. C. CARLson, The Logarithmic Mean, MAA Monthly 79 (1972), 615-618.

[5] P. Kahlig, J. Matkowski, Functional equations involving the logarithmic mean, Z. Angew. Math. Mech. 76 (1996), no. 7, 385-390.

[6] J. M. Kay, R. M. Nedderman, An Introduction to Fluid Mechanics and Heat Transfer, Cambridge University Press, 1979.

[7] F. Kreith, Principles of Heat Transfer, Harper \& Row, New York, 1973.

[8] J. Matkows KI, Iterations of mean-type mappings and invariant means, Ann. Math. Siles. 13 (1999), 211-226.

[9] J. MatKows KI, Invariant and complementary quasi-arithmetic means, Aequationes Math. 57 (1999) $87-107$.

[10] J. MatKows KI, Iterations of the mean-type mappings, (ECIT'08), (A. N. Sharkovsky, I. M. Sushko Eds.), Grazer Math. Ber., Bericht Nr. 354 (2009), 158-179.

[11] J. MATKOWSKI, Iterations of the mean-type mappings and uniqueness of invariant means, Annales Univ. Sci. Budapest., Sect. Comp. 41 (2013), 145-158.

[12] A. WiTKOWS KI, An even easier proof of monotonicity of Stolarsky means, Kragujevac J. Math. 35 (3) $2011,447-450$. 